\title{
Mechanical Properties and Eigenfrequencies of Graphene Sheets
}

\author{
Pavol Lengvarský ${ }^{1, *}$ and Jozef Bocko ${ }^{1}$ \\ ${ }^{1}$ Technical University of Košice, Faculty of Mechanical Engineering, Department of Applied Mechanics and Mechanical Engineering, Letná 9, 04200 \\ Košice, Slovakia
}

\begin{abstract}
The Membrane stiffness, Young's moduli, Poisson's ratio and eigenfrequencies are investigated in this paper. Graphene sheet is modelled by beam elements. The properties of these elements are directly derived from interatomic potentials. Graphene sheet is modelled with commercial finite element code and the boundary conditions are applied in two different directions. From these simulations the membrane stiffness C, Young's modulus $Y$ and the Poisson's ratio $\mu$ of the graphene sheets are obtained. Then the first twenty eigenfrequencies of the graphene sheets are investigated.
\end{abstract}

Keywords: graphene sheet; beam element; Young's modulus; eigenfrequencies.

\section{Introduction}

Graphene is a two-dimensional hexagonal (honeycomb) lattice made of carbon atoms which size is in nanometers. Materials in nanometers are widely investigated for their extraordinary properties. Researches study their mechanical properties and their applications in classic materials. Young's modulus, tensile or compressive strength, eigenfrequencies and buckling are investigated by number of researchers. Intensive research is also oriented to the application of nanostructures in electrical and chemical engineering, as well as in biological sciences [1].

\section{Molecular Mechanics}

Membrane stiffness, Young's modulus and Poisson's ratio is calculated from deformation of graphene sheet but at the beginning the knowledge of beam element parameters is necessary. For this sake we define connection between molecular mechanics and structural mechanics [2], [3]. Molecular mechanics approach is commonly used for modeling graphene sheets. However, this method and supercomputers can simulate the behavior of carbon nanotubes only with small lengths (several micrometers). Determining of solution is also a considerably time-consuming process. Therefore, using FEM to describe the behaviors of graphene sheets is better than using molecular mechanics. Graphene sheets modeling can be realized by different types of finite elements, i.e., truss, beam, linear and nonlinear spring, and shell elements.

In molecular mechanics graphene sheet can be regarded as a large molecule consisting of carbon atoms and the atomic nuclei as material points. Motions of atomic nuclei are regulated by a force field, which is generated by electron-nucleus interactions and nucleus-nucleus interaction and the force field is expressed in the 
form of steric potential energy [4]. The total steric potential energy [5]-[10] omitting the electrostatic interaction, is a sum of energies due to valence or bonded interactions and non-bonded interactions and is expressed as

$$
U_{\text {total }}=\sum U_{r}+\sum U_{\theta}+\sum U_{\phi}+\sum U_{\omega}+\sum U_{v d w},
$$

where $U_{r}, U_{\theta}, U_{\phi}, U_{\omega}, U_{v d w}$ are a bond stretch interaction, a bond angle bending, a dihedral angle torsion, an improper (out of plane) torsion, a nonbonded van der Waals interaction, respectively. Representations of these interactions are given in Fig. 1 [6], [11]-[12].
Dihedral angle torsion

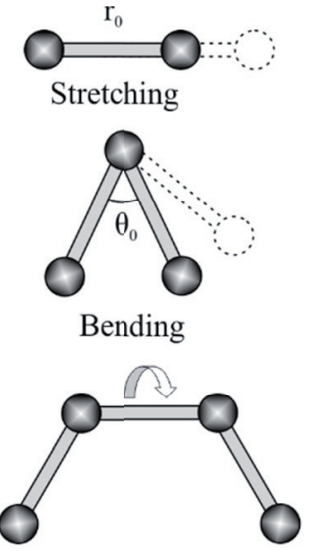

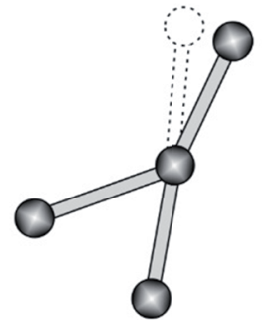

Out of plane torsion

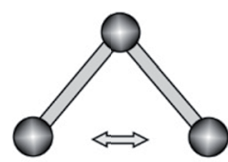

van der Waals
Fig. 1: Interatomic interactions in molecular mechanics [2].

The main contributions to the total steric energy come from the first four terms of equation (1). Under the assumption of small deformation, the harmonic approximation is adequate for describing the energy [5]. By adopting the simplest harmonic forms and merging dihedral angle torsion and outof-plane torsion into a single equivalent term, we can write relations

$$
\begin{aligned}
& U_{r}=\frac{1}{2} k_{r}\left(r-r_{0}\right)^{2}=\frac{1}{2} k_{r}(\Delta r)^{2}, \\
& U_{\theta}=\frac{1}{2} k_{\theta}\left(\theta-\theta_{0}\right)^{2}=\frac{1}{2} k_{\theta}(\Delta \theta)^{2}, \\
& U_{\tau}=U_{\phi}+U_{\omega}=\frac{1}{2} k_{\tau}(\Delta \phi)^{2},
\end{aligned}
$$

where $k_{r}, k_{\theta}, k_{\tau}, \Delta r, \Delta \alpha, \Delta \phi$ are the bond stretching force constant, bond angle bending force constant, torsional resistance, the bond stretching increment, the bond angle change and the angle change of bond twisting, respectively [2]-[3].

To calculate the elastic moduli of beam elements we determine relations between the sectional stiffness parameters in structural mechanics and the force constants in molecular mechanics.

The sections of beams which represent carboncarbon bonds are assumed as identical, circular and moments of inertia are $I_{x}=I_{y}=I$. Than we get three stiffness parameters EA,EI and GJ from linkage among the energy terms in molecular mechanics and continuum mechanics as

$$
\begin{aligned}
& U_{A}=\frac{1}{2} \int_{0}^{L} \frac{N^{2}}{E A} d L=\frac{1}{2} \frac{N^{2} L}{E A}=\frac{1}{2} \frac{E A}{L}(\Delta L)^{2}, \\
& U_{M}=\frac{1}{2} \int_{0}^{L} \frac{M^{2}}{E I} d L=\frac{2 E I}{L} \alpha^{2}=\frac{1}{2} \frac{E I}{L}(2 \alpha)^{2}, \\
& U_{T}=\frac{1}{2} \int_{0}^{L} \frac{T^{2}}{G J} d L=\frac{1}{2} \frac{T^{2} L}{G J}=\frac{1}{2} \frac{G J}{L}(\Delta \beta)^{2},
\end{aligned}
$$

where $U_{A}$ is the strain energy of a uniform beam of length $L$ subjected to axial force $N, \Delta L$ is axial elongation, $U_{M}$ is the strain energy of a uniform beam under bending moment $M, \alpha$ is the rotational angle at the ends of the beam, $U_{T}$ is the strain energy of a uniform beam under tension $T$ and $\Delta \beta$ is the relative rotation between the ends of the beam (Fig. 2) [2]-[3].

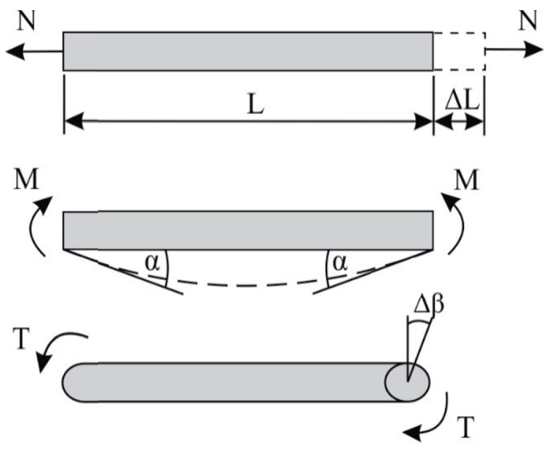

Fig. 2: Tension, beneding and torsion of an element.

Thus by comparing equations (2)-(4) and (5)-(7) we get terms

$$
\frac{E A}{L}=k_{r}, \frac{E I}{L}=k_{\theta}, \frac{G J}{L}=k_{\tau},
$$


Then beam element (Fig. 3) is adopted and its elastic properties for further analysis are

$d=4 \sqrt{\frac{k_{\theta}}{k_{r}}}, E=\frac{k_{r}^{2} L}{4 \pi k_{\theta}}, G=\frac{k_{r}^{2} k_{\tau} L}{8 \pi k_{\theta}^{2}}$,

where constants $k_{r}, k_{\theta}, k_{\tau}, L$ are $k_{r}=6.52 \cdot 10^{-7} \mathrm{Nnm}^{-1}$, $k_{\theta}=8.76 \cdot 10^{-10} \mathrm{Nnmrad}^{-1}, k_{\tau}=2.78 \cdot 10^{-10} \mathrm{Nnmrad}^{-2}$

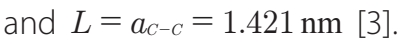

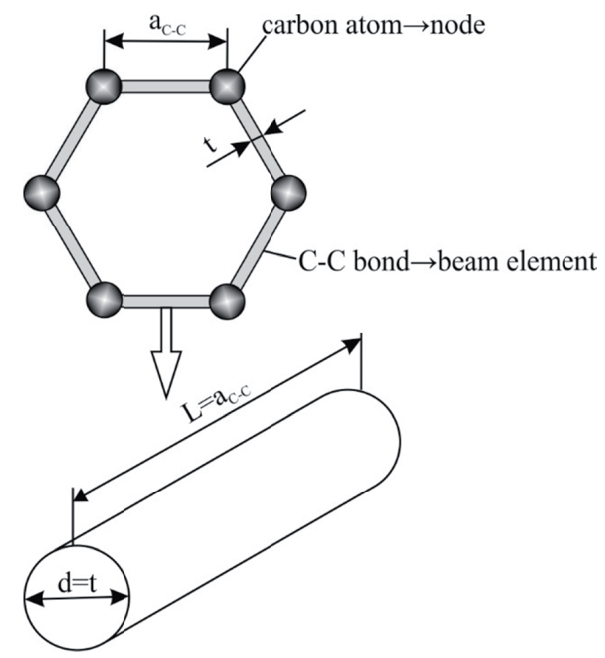

Fig. 3: Space frame structure.

Terms in (9) are calculated and we get diameter $d=0.147 \mathrm{~nm}$, elastic moduli $E=5.4875 \mathrm{TPa}$ and $G=0.871 \mathrm{TPa}$ for beam elements.

\section{Structural Analysis}

The numerical computations are accomplished by commercial program Ansys. Graphene sheet is modelled as frame structure by beam elements which have had parameters mentioned above. Carbon atoms are considered to be nodes in structure and the nodes are joined by beam elements. The structural response of the graphene sheets under axial loads is analyzed. To simulate the uniaxial load, one side of the graphene sheet is restrained in axial direction with lateral displacements being allowed. The graphene sheets are stretch by applying an axial force on the opposite side. The finite element analysis is performed for two different boundary conditions (graphene sheet rotated by $90^{\circ}$ ) and from this we get two different chiralities of graphene sheets. From these simulations the membrane stiffness $C$ Young's modulus $E$ and the Poisson's ratio $\mu$ of the graphene sheets are obtained.

The membrane stiffness $C$ is obtained from equation

$C=Y t$,

where $Y$ is the Young's modulus and $t$ is the thickness of graphene sheet. The Young's modulus is ratio of normal stress to normal strain obtained from equation

$Y=\frac{\sigma}{\varepsilon}=\frac{F / A_{0}}{\Delta H / H_{0}}$,

where $F, A_{0}, \Delta H, H_{0}$ are total force applied on graphene sheet, the cross-section of graphene sheet, the elongation of graphene sheet and the initial length of graphene sheet, respectively. $A_{0}$ is equal $W_{0} \cdot t$, where $W_{0}$ is the initial width of graphene sheet. When equation (11) is substituted to equation (10) we get membrane stiffness independent on thickness of graphene sheet.

The Poisson's ratio of graphene sheet is calculated from equation

$\mu=-\frac{\varepsilon_{W}}{\varepsilon_{H}}=-\frac{\Delta W / W_{0}}{\Delta H / H_{0}}$,

where $\Delta W$ is constriction of graphene sheet.

When the thickness $t$ of graphene sheet is the same as diameter $d$ of the beam element [11], then Young's moduli and Poisson's ratios are computed and some results are given in Table 1.

All computed Young's moduli are shown in Fig. 4. It is clear that Young's moduli of graphene sheets increase slightly with the size of the sheets and depends on the chirality.

Poisson's ratio for graphene sheet is computed from equation (12) and corresponding charts are shown in Fig. 5.

Some authors use in equation (11) in term $A_{0}$ for $t$ value $0.34 \mathrm{~nm}$, which is the interlayer spacing of graphite. In Table 2 are given new values of Young's moduli of graphene sheet and corresponding dependencies.

Variation of the Young's modulus is shown in Fig. 6.

Finally, in Fig. 7 is shown membrane stiffness, which is independent on thickness of graphene sheet. 
Table 1: Young's moduli for different dimensions of graphene sheets.

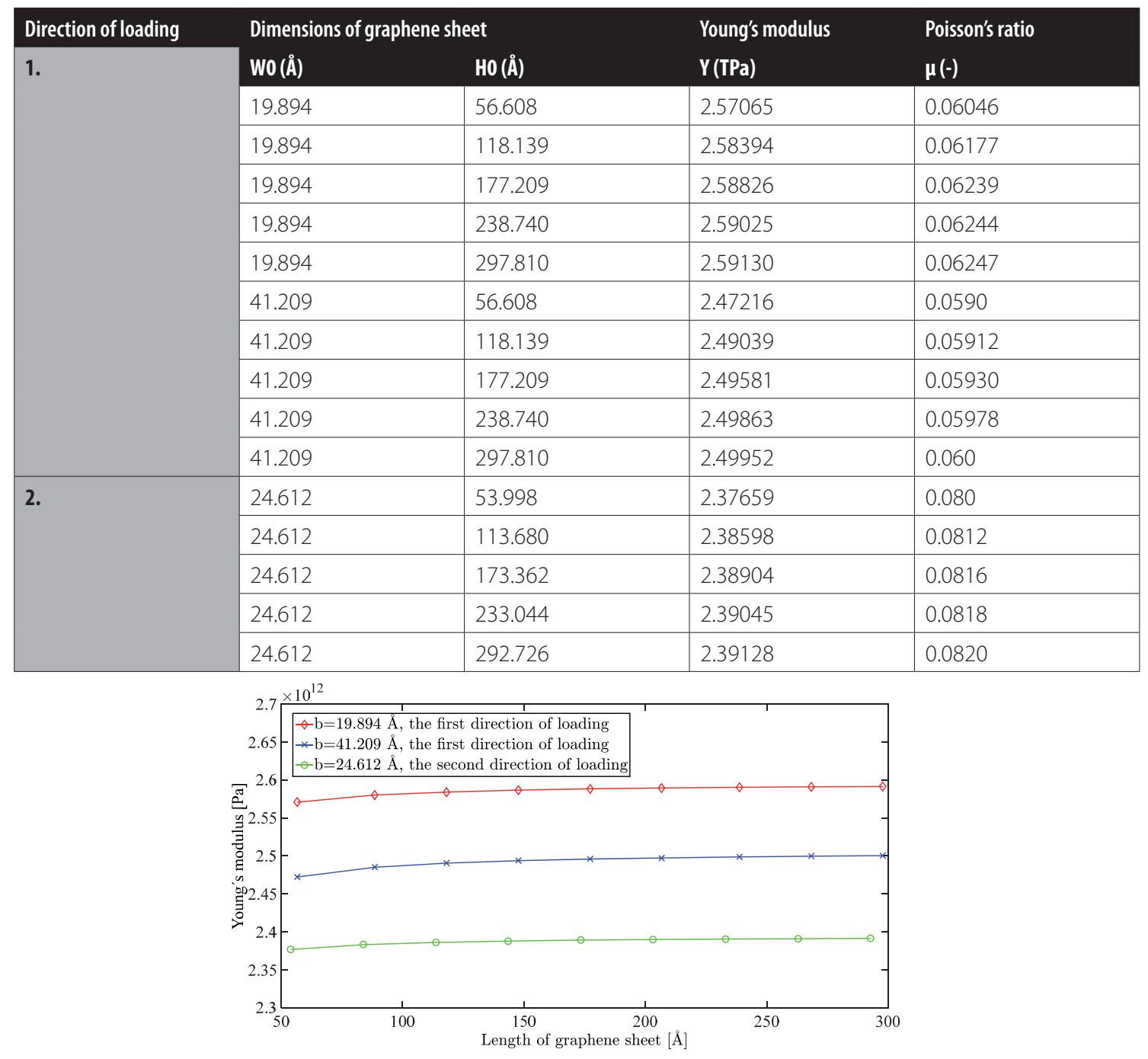

Fig. 4: Variation Young's modulus with change length of graphene sheet.

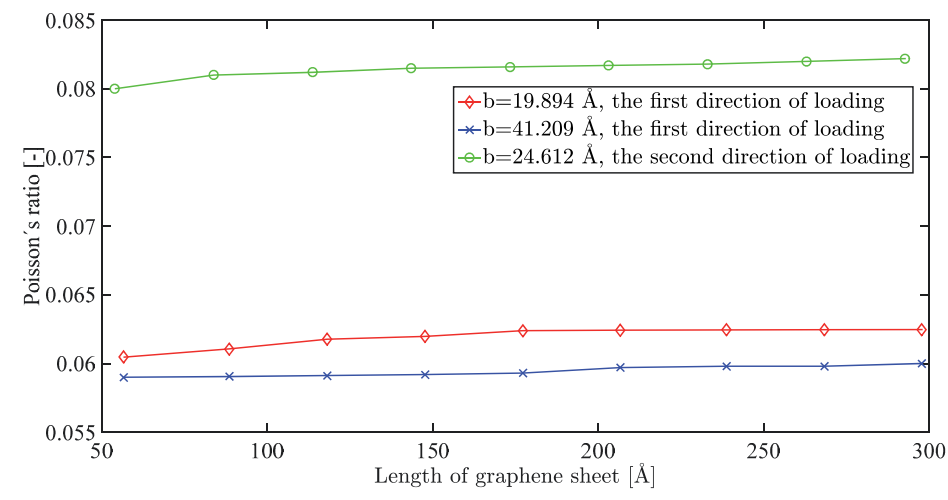

Fig. 5: Variation Poisson's ratio with change length of graphene sheet. 
Table 2: Young's moduli for different dimensions of graphene sheets.

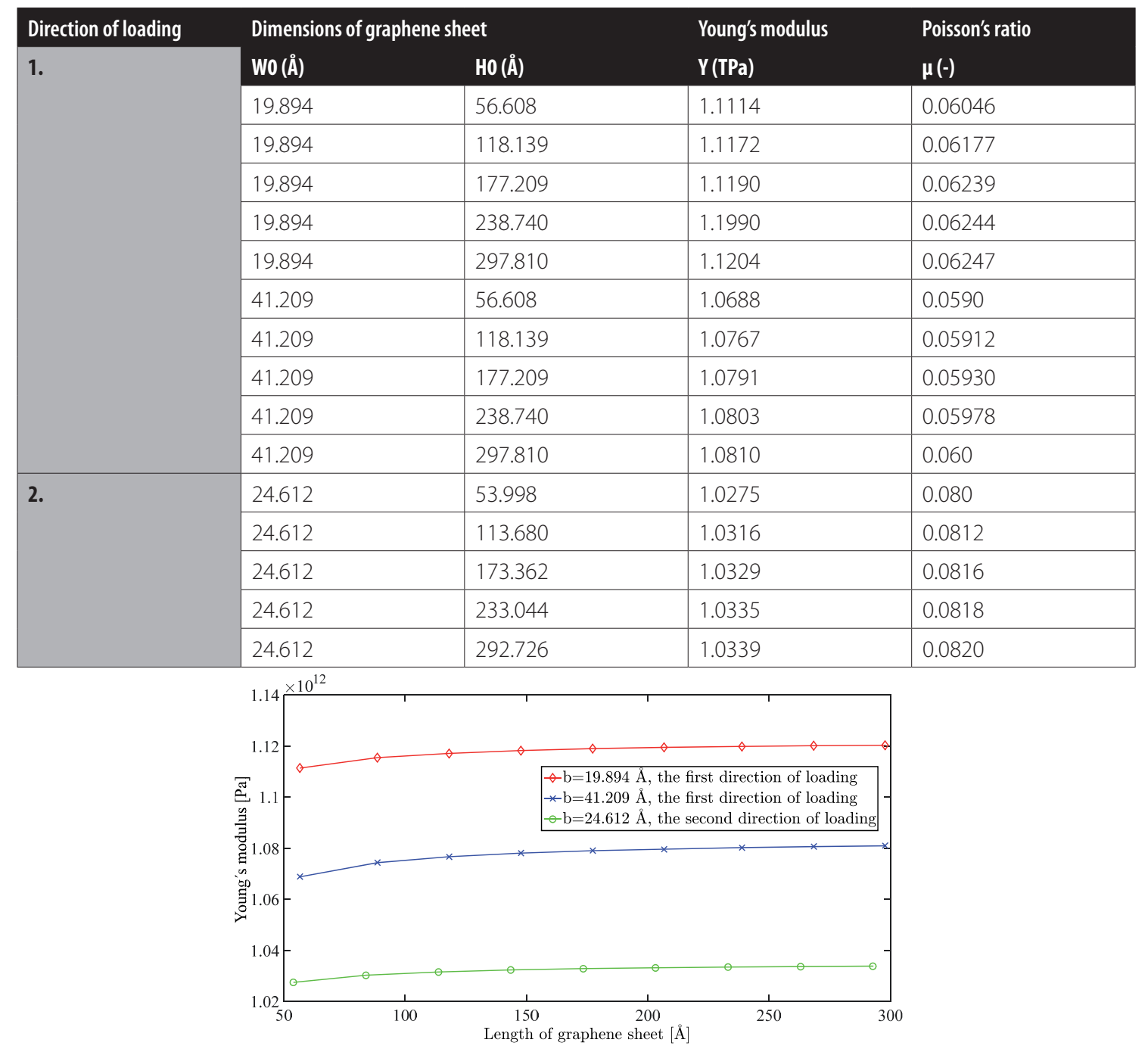

Fig. 6: Variation Young's modulus with change length of graphene sheet.

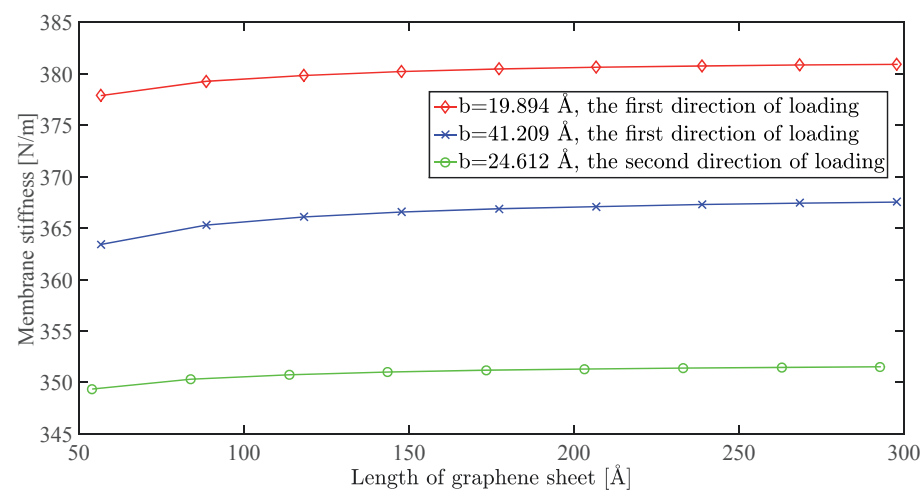

Fig. 7: Variation membrane stiffness with change length of graphene sheet. 


\section{Modal analysis}

The eigenfrequencies are very important parameters of those structures because their measurement can serve as a base for the determination of elastic properties, e.g., Young's modulus of homogenized materials. Modal analysis is performed by commercial code in Ansys APDL. Carbon atoms are joined by beam elements with mass density $\rho=2250 \mathrm{~kg} / \mathrm{m}^{3}$ and carbon atoms are considered to be mass elements with mass $2 \cdot 10^{-26} \mathrm{~kg}$. The two limitless boundary conditions are considered i.e. all edges of graphene sheet is free (FFFF) and all edges are clamped (CCCC). The first twenty eigenfrequencies of the graphene sheets with length $30 \AA, 300 \AA$ and $900 \AA$ are computed and these are shown in Fig. 8 - Fig. 10.

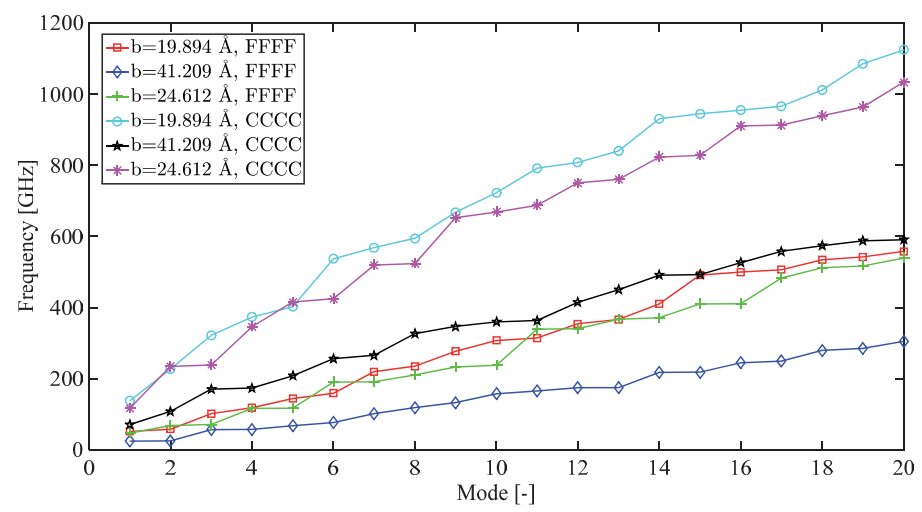

Fig. 8: Eigenfrequencies of graphene sheet with length $30 \AA$.

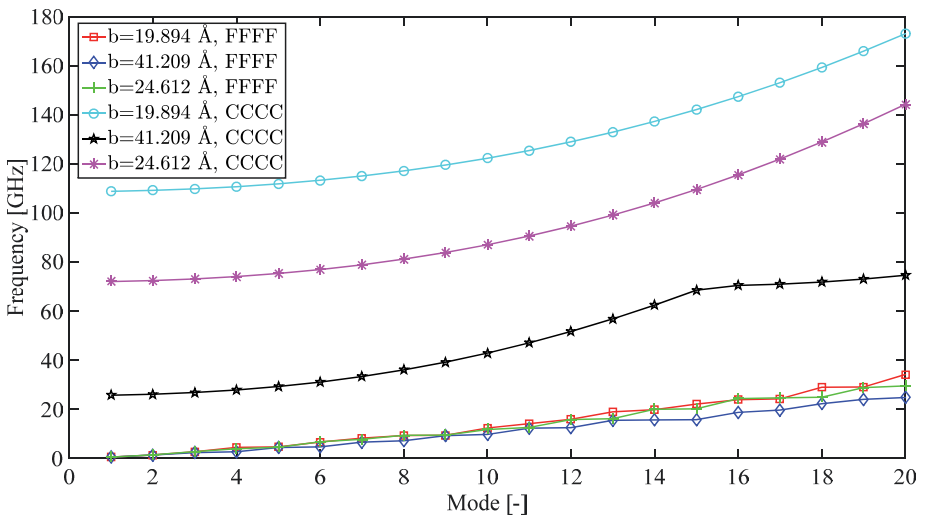

Fig. 9: Eigenfrequencies of graphene sheet with length $300 \AA$.

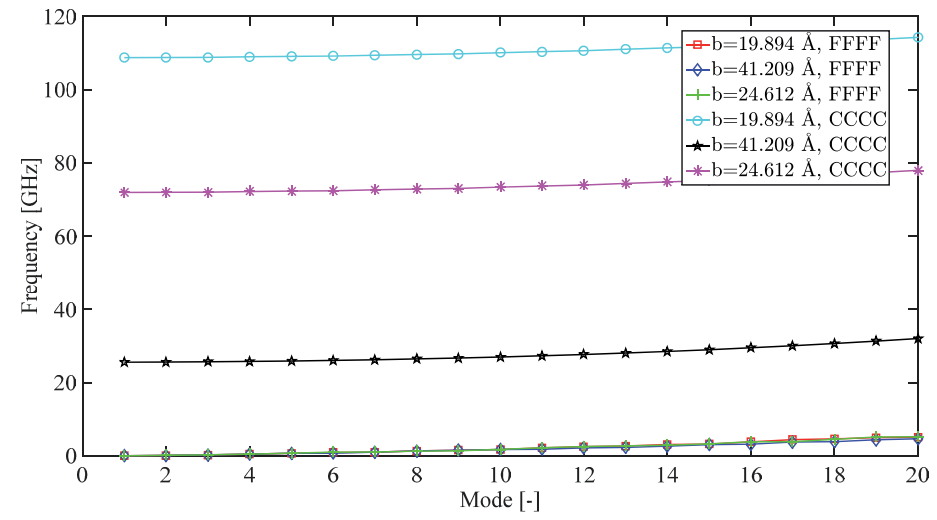

Fig. 10: Eigenfrequencies of graphene sheet with length $900 \AA$. 


\section{Conclusions}

A linkage between molecular mechanics and structural mechanics has been proposed. For the computation is used finite element model with beam elements of proposed diameter and elastic properties (Young's moduli). This model is based only on beam elements that represent C-C bonds and joints that represent carbon atoms. The beam connects individual atoms (joints). Analysis has been performed on graphene sheets with different dimensions like width, length and with two different loading directions. The same boundary conditions were applied consecutively to two mutually perpendicular directions.

This model was used for evaluation of Young's modulus and Poisson's ratio of graphene sheet. Young's modulus is affected by dimensions, direction of loading of graphene sheet and Poisson's ratio is affected by direction of loading of graphene sheet. Young's modulus and Poisson's ratio increase slightly with increasing length of graphene sheet. Magnitudes of Young's modulus and Poisson's ratios are comparable with results published by several researchers from theoretical, numerical (molecular, structural simulations) and experimental measurements.

The eigenfrequencies of these graphene sheets are investigated. The mass and the mass density of the carbon atoms and connections are assigned. For the totally fixed graphene sheets are eigenfrequencies two times higher and too depend on chiralities of these graphene sheets.

\section{Acknowledgments \\ This article is created with support of VEGA grant project VEGA 1/0731/16.}

\section{References and Notes}

[1] Marenić, E., Ibrahimbegovic, A., Sorić, J. Guidault, P.A., (2013). Homogenized elastic properties of graphene for small deformations. Materials, 6, 3764-3782.

[2] Li, Ch., Chou, T., (2003). A structural mechanics approach for the analysis of carbon nanotubes. International Journal of Solids and Structures, 40, 2487-2499.

[3] Tserpes, K.I., Papanikos, P., (2005). Finite element modelling of single-walled carbon nanotubes. Composites: Part B, 36, 468-477.

[4] Machida, K., (1999). Principles of Molecular Mechanics, Kodansha and John Wiley \& Sons Co-publication, Tokyo.

[5] Gelin, B.R., Molecular modeling of polymer structures and properties, Hanser/Gardner Publishers, Cincinnati. 1994.

[6] Mayo, S.L., Olafson, B.D., Goddard, W.A., (1990). Dreiding-a generic force-field for molecular simulations. Journal of Physical Chemistry, 94, 8897-8909.

[7] Cornell, W.D., Cieplak, P., Bayly, C.I., et al., (1995). A second generation force-field for the simulation of proteins, nucleic-acids, and organic-molecules. Journal of American Chemical Society, 117, 5179-5197.

[8] Brenner, D.W., (1990). Empirical potential for hydrocarbons for use in simulating the chemical vapor deposition of diamond films. Physical Review B, 42, 9458.

[9] Rappe, A.K., Casewit, C.J., Colwell, K.S., et al., (1992). A full periodic-table force-field for molecular mechanics and molecular dynamics simulations. Journal of American Chemical Society, 114, 10024-10035.

[10] Ru, C.Q., (2000). Effective bending stiffness of carbon nanotubes. Physical Review B, 62, 9973-9976.

[11] Saito, S., Dresselhaus, D., Dresselhaus, M.S., (1998). Physical Properties of Carbon Nanotubes, Imperical College Press, London.

[12] Thostenson, E.T., Chunyu, L., Chou, T.W. (2005). Nanocomposites in context. Composite Science and Technology, 65, 491-516.

\section{Biographical notes}

Pavol Lengvarský, Ing., PhD. (1988) is an assistant professor of applied mechanics on the Department of Applied Mechanics and Mechanical Engineering. In 2017 he received doctoral degree in the field of applied mechanics. He is author more than 30 publications in journals and conference proceedings in Slovakia and abroad. His professional and scientific interests are oriented to the finite element method, nonlinear mechanics, shell theory, modelling of nanoparticles behavior by means of classical continuum mechanics.

Jozef Bocko, prof., Ing., CSc. (1959) is a professor of applied mechanics. He is author of 2 monographs, 9 university textbook and more than 200 publications in journals and conference proceedings in Slovakia and abroad. He is author and co-author of important projects and engineering works. His professional and scientific interests are oriented to the finite element method, nonlinear mechanics, shell theory, application of Lie groups in mechanics. He is a member of Scientific council of the Faculty of Mechanical Engineering, Technical University of Košice and member of council of Slovak Association of Mechanics by Slovak Academy of Sciences. 